\title{
Trypanosoma cruzi: Kinetics of Metacyclogenesis in Adult and Nymphal Panstrongylus megistus
}

\author{
JOSEPH PIESMAN \\ Department of Tropical Public Health, Harvard School of Public Health, 665 Huntington Avenue, \\ Boston, Massachusetts 02115, U.S.A.
}

AND

ITALO A. SHERLOCK

Centro de Pesquisas Goncalo Moniz, Rua Waldemar Falcao 121, Brotas, 40.000 Salvador, Bahia, Brazil

(Accepted for publication 6 November 1984)

\begin{abstract}
Piesman, J., ANd Sherlock, I. A. 1985. Trypanosoma cruzi: Kinetics of metacyclogenesis in adult and nymphal Panstrongylus megistus. Experimental Parasitology 59, 231-238. Adult triatomine insects, Panstrongylus megistus, naturally infected with the protozoa, Trypanosoma cruzi, contained significantly more metacyclic trypomastigote forms in their digestive tracts than did $P$. megistus nymphs. Metacyclics were scarce in all stages of triatomines fed once on infected mammalian hosts and subsequently starved. In contrast, triatonines fed repededly on infected nimmnalian hosts developed prolific $T$. cruzi infections. Repeatedly fed adults contained $14 \times$ more metacyclics than similarly fed nymphs. Nutritional factors appear to play a key role in regulating metacyclogenesis. Control campaigns designed to alter the age structure of populations of triatomines must take notice of the high transmission potential of the adult stage. (c) 1985 Academic Press, Inc.

INDEX Descriptors: Trypanosoma cruzi; Protozoa, parasitic; Hemoflagellate; Chagas' disease; Metacyclogenesis; Nutritional factors; Trypomastigotes; Panstrongylus megistus; Triatomine; Insect; Guinea pig.
\end{abstract}

\section{INTRODUCTION}

The development of Trypanosoma cruzi within triatomine vectors has long been known. Brumpt (1912) originally recognized that the stage infectious to vertebrates, metacyclic trypomastigotes, is present in the hindgut of the insect. Moreover, he realized that transmission occurred on contact with contaminated feces. Dias (1934) later confirmed these findings. The process by which $T$. cruzi transforms into the metacyclic stage, "metacyclogenesis" (Zeledon et al. 1977), has been quantified in vitro (Fernandes et al. 1969; Pan 1978a; Sher et al. 1983; Ucros et al. 1983). In contrast, studies of metacyclogenesis within triatomines have been principally descriptive (Brack 1968; Brener 1972; Zeledon et al. 1984). Attempts have been made to measure the number of metacyclic trypomastigotes $(=$ metacyclics $)$ in triatomines (Mshelbwala and Ormerod 1973; Neal and McHardy 1977; Urdancta-Morales and Rueda 1977; Perlowagora-Szumlewicz and Muller 1982), but information concerning the kinetics of metacyclogenesis in vivo is lacking.

Torres (1915) was the first to note that infection rates of $T$. cruzi in naturally infected adult triatomines exceeded those of nymphal triatomines. Others have confirmed this observation (Minter 1978; Zarate et al. 1980). However, the density of trypanosomes in naturally infected adult and nymphal triatomines has not been com- 
pared. Wood (1960) observed that fecal droplets of laboratory-infected adult Triatoma protacta contained more metacyclic trypomastigotes than similar droplets from nymphal $T$. protracta. In this study, we determined the density of metacyclic trypomastigotes present in different stages of naturally infected Panstrongylus megistus. In addition, we examined the factors which control metacyclogenesis in nymphal and adult triatomines.

\section{MATERials AND MeTHOdS}

The CA 31 strain of Trypanosoma cruzi, used to experimentally infect triatomines, was isolated from four infected Panstrongylus megistus collected from an infested house in Castro Alves, Bahia, Brazil, in May, 1982. A pooled fecal sample from these triatomines was inoculated intraperitoneally into a guinea pig. For routine maintenance of the strain, parasites were passaged alternately between guinea pigs and triatomines at 1 -month intervals. Triatomines were allowed to feed on guinea pigs containing 1-10 trypomastigotes $/ \mathrm{mm}^{3}$ blood. Guinea pigs were inoculated intraperitoncally with the pooled feces of four to six infected $P$. megistus containing about $10^{6}$ flagellates.

$P$. megistus were obtained from colonies maintained at the Centro de Pesquisas Goncalo Moniz, Salvador, Bahia, Brazil, for up to 10 years. Colonies were maintained by weekly feedings on chickens. These colonies originate from $P$. megistus collected in the state of Bahia, Brazil.

Triatomines were starved for 15-30 days after molting before being fed on infected guinea pigs that earlier had been inoculated intraperitoneally with $10^{6}$ flagellates harvested from $P$. megistus feces. Four to five weeks after inoculation, bugs were fed on animals with parasitemias varying between 2.0 and 6.6 trypomastigotes $/ \mathrm{mm}^{3}$ blood. Parasitemia in hosts was determined by counting the number of trypanosomes in $5 \mathrm{~mm}^{3}$ of blood placed onto a microscopic slide and examined under a phase-contrast objective at $400 \times$ magnification. Bugs in plastic cups were held in contact with shaved guinea pig skin for $1 \mathrm{hr}$ during each experimental feeding. Infected triatomines were held at room temperature $(25-30 \mathrm{C}$; $70-90 \%$ relative humidity).

We determined the number of flagellates present in individual triatomine digestive tracts using the techniques of Miles et al. (1975). A $150-\mathrm{mm}^{3}$ drop of phosphate-buffered saline was placed into $500-\mathrm{mm}^{3}$ wells of plastic microtiter plates (Sterlin Co., Surrey, England, UK). The entire contents of a digestive tract was placed in a well and thoroughly macerated be- tween the tips of fine dissecting forceps. The fecal suspension was withdrawn for measurement in a 100 $\mathrm{mm}^{3}$ Wiretrol pipet (Drummond Scientific Co. Broomall, PA, USA) and graduated into 50 equal divisions, and the volume was measured to the nearest $2 \mathrm{~mm}^{3}$ prior to transfer to another well. A $15-\mathrm{mm}^{3}$ sample of feces was then transferred to a microscope slide and examined under phase-contrast at $400 \times$ magnification. If flagellates were detected, the density of flagellates (no. flagellates $/ \mathrm{ml}$ fecal solution) was determined with a hemocytometer. If the parasite suspension was dense, a preliminary 1:10 dilution in phosphate-huffered saline was made before counting. If only a few parasites were present, a $15 \mathrm{~mm}^{3}$ sample was placed on a microscopic slide under a $22 \times 22$ mm coverslip and the total number of flagellates was determined. We determined the number of parasites present in each bug as follows: Number of flagellates/ milliliter fecal solution $\times$. volume fecal solution $=$ Number of flagellates per bug. In order to test the accuracy of the assay, we added known quantities of cultured trypanosomes $\left(10^{3}\right.$ to $\left.10^{6}\right)$ to diluted bug feces. The number of parasites detected in the assay was generally slightly less than the known quantity added (mean $=-6 \%$ in 40 trials).

Two methods were used to quantitate the motile parasite stages present in triatomine digestive tracts. Epimastigotes were distinguished from metacyclic trypomastigotes on the basis of motility in fresh $15-\mathrm{mm}^{3}$ fecal samples, viewed with a phase-contrast objective at $400 \times$ magnification. In addition, a $10-\mathrm{mm}^{3}$ fecal sample was spread on a slide, dried, and stained with Wright-Giemsa stain as previously described (Pan 1978a). Stained slides were examined at $1000 \times$ magnification. Epimastigotes were distinguished from metacyclic trypomastigotes on the basis of morphology (Hoare and Wallace 1966). Nonmotile amastigotes or "sphaeromastigotes" were not counted. The phase-contrast and Giemsa stain methods were applied to fecal samples collected from 50 naturally infected $P$. megistus. All preparations were examined for $20 \mathrm{~min}$ or until 200 parasites had been counted. The mean proportion of metacyclics was similar in both methods (phase-contrast, 32.9\%; Giemsa stain, 28.6\%; $r=0.94, P<0.001$ ). Thereafter, only stained preparations were examined.

Triatomines were collected from 247 houses in Castro Alves, Bahia, Brazil, where Chagas' disease is endemic (Mott et al. 1976, 1978; Piesman et al. 1983). $P$. megistus is the only triatomine species commonly infesting houses. Bugs were collected from the walls and beds of infested houses without pyrethrum application. Captured triatomines were taken to the laboratory, maintained at room temperature, and examined for evidence of infection within 6 days after collection. Triatomine collections were made between August, 1981, and July, 1982. 
TABLE I

Prevalence of Trypanosoma cruzi Infection According to Stage in Nymphal and Adult Panstrongylus megistus Collected in Castro Alves, Bahia, Brazil

\begin{tabular}{ccc}
\hline Stage & $\begin{array}{c}\text { No. } \\
\text { examined }\end{array}$ & $\begin{array}{c}\text { Percentage } \\
\text { of bugs } \\
\text { infected }\end{array}$ \\
\hline 1 & 110 & 2.7 \\
2 & 144 & 8.3 \\
3 & 196 & 21.4 \\
4 & 171 & 26.9 \\
5 & 228 & 31.6 \\
$\mathrm{M}^{a}$ & 99 & 44.4 \\
$\mathrm{~F}^{b}$ & 127 & 47.2 \\
\hline
\end{tabular}

a Male.

${ }^{b}$ Female.

\section{RESULTS}

A total of 1075 Panstrongylus megistus were collected from 247 houses in Castro Alves. About 25\% were infected (Table I). The proportion infected was related to age, being greatest in adult females (47\%) and least in first stage nymphs (3\%). The prevalence of infection in adults (46\%) was sig- nificantly greater than in nymphs $(21 \%)\left(\chi^{2}\right.$ $=59.8, P<0.001$ ).

We determined the number of flagellates and metacyclic trypomastigotes in 228 naturally infected $P$. megistus (Fig. 1). The overall number of flagellates and metacyclics per insect increased with age; adults had significantly more flagellates $(t=9.02$, $P<0.001)$ and metacyclics $(t=9.24, P<$ $0.001)$ than did nymphs. Since adults are generally larger than nymphs, we weighed all triatomines (to the nearest $0.1 \mathrm{mg}$ ) before examination and computed the density of flagellates and metacyclics per milligram insect body weight. While flagellate density per milligram was only slightly greater in adults than in nymphs, the metacyclic density was significantly $(t=8.06, P<0.001)$ higher in adults. Moreover, a significantly greater proportion of parasites in adult bugs were in the metacyclic stage $(\overline{\mathrm{x}}=37.5 \%$, $\mathrm{SD}=23.6)$ as compared to parasites in nymphs $(\overline{\mathrm{x}}=18.7 \%, \mathrm{SD}=22.3)(t=5.89$, $P<0.001$ ).

The flagellate and metacyclic density in individual bugs of different stages, each fed

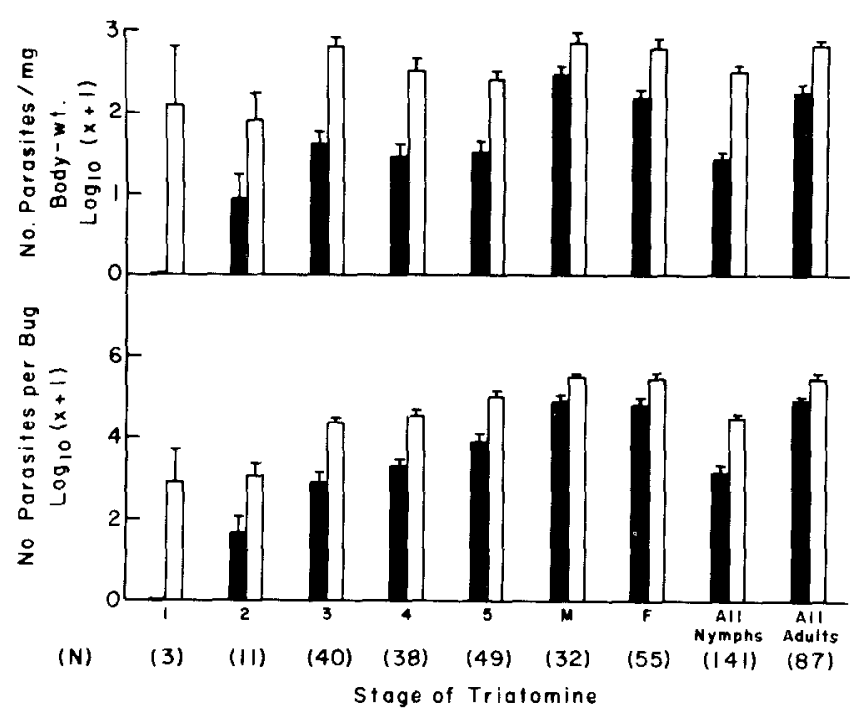

FIG. 1. Numbers of Trypanosoma cruzi flagellates in naturally infected Panstrongylus megistus captured in houses in Castro Alves, Bahia, Brazil. Light bars represent mean number of flagellates. Dark bars represent mean number of metacyclic trypomastigote forms $( \pm \mathrm{SE})$. 
once on a Trypanosoma cruzi-infected guinea pig and starved for 1 month, was measured (Fig. 2). There was no difference in the number of flagellates among the various stages. Adults had slightly higher metacyclic densities overall, but third-stage nymphs contained more metacyclics than did adults. Thus, metacyclogenesis in starved adults and nymphs was similar.

In a subsequent experiment, the effect of multiple infectious feedings was evaluated. Fifty adult female and 50 fourth-stage nymphal $P$. megistus were allowed to feed on infected guinea pigs at weekly intervals over a 2-month period. At the end of this period, surviving triatomines were examined. Flagellate density was similar in females and fourth-stage nymphs (Table II). However, significantly more metacyclics were present ( $t=5.47, P<0.001$ ) in adults and the density of metacyclics per $\mathrm{mg}$ was greater in adults $(t=4.64, P<0.001)$. The mean proportion of parasites in the metacyclic stage was $29.6 \%( \pm 16.1$ SD) in adults, as opposed to $11.6 \%( \pm 10.4 \mathrm{SD})$ in nymphs $(t=6.07, P<0.001)$.

Individual triatomines were weighed before and after each weekly feeding during this experiment. The ingestion pattern in adults and nymphs differed markedly. During the 8 weeks of feedings, adults ingested more blood ( $\overline{\mathrm{x}}=118.9 \mathrm{mg}$ per week) than did fourth-stage nymphs $(\bar{x}=57.2$ $\mathrm{mg}$ ). Moreover, the amount of blood digested from one week to the next was much greater in adults $(\overline{\mathrm{x}}=88.8 \%$ of prior weeks bloodmeal) than in nymphs $(67.5 \%)$. Consequently, the mean number of trypanosomes ingested by adults per week (358.2) was more than twice that ingested by nymphs (167.5).

The pattern of metacyclogenesis in adult and nymphal triatomines was examined. The number of flagellates and metacyclics in 12 adults and 12 nymphs fed weekly on infected guinea pigs was determined at 2-week intervals. While there were no metacyclics present in nymphs at 2 weeks after initial bloodmeal, metacyclics were present in adults (Fig. 3). The density of metacyclics increased rapidly from Weeks 2 to 4 in adults. In contrast, the density of metacyclics rose gradually in nymphs during Weeks 2 to 8 . Adults always contained more metacyclics.

The resistance of adult and nymphal $P$.

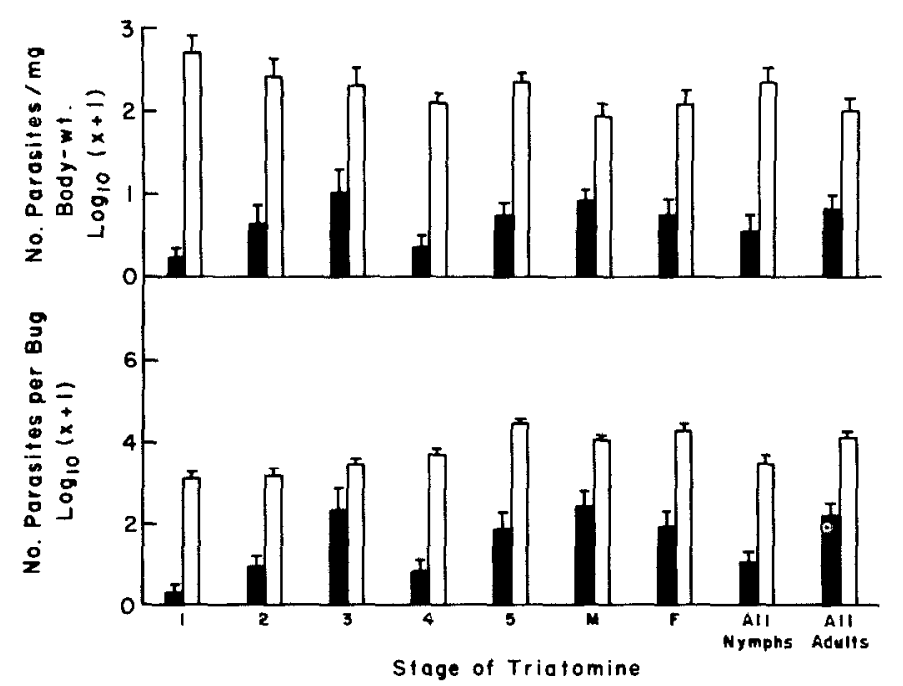

FIG. 2. Numbers of Trypanosoma cruzi in the triatomine, Panstrongylus megistus, fed once on an infected guinea pig. Triatomines were examined 1 month after feeding. A total of 25 infected triatomines of each stage were examined. Flagellate and metacyclic density represented as in Fig. 1. 
TABLE II

Number of Trypanosoma cruzi Flagellates and Metacyclics in Panstrongylus megistus Examined Two Months after Initial Feeding

\begin{tabular}{cccccc}
\hline Stage & $\begin{array}{c}\text { No. } \\
\text { bugs }\end{array}$ & $\begin{array}{c}\text { No. } \\
\text { flagellates } \\
\text { per bug }\end{array}$ & $\begin{array}{c}\text { No. } \\
\text { metacyclics } \\
\text { per bug }\end{array}$ & $\begin{array}{c}\text { No. } \\
\text { flagellates } \\
\text { per } \mathrm{mg} \\
\text { body wt }\end{array}$ & $\begin{array}{c}\text { No. } \\
\text { metacyclics } \\
\text { per mg } \\
\text { body wt }\end{array}$ \\
\hline $\mathrm{F}^{a}$ & $41^{b}$ & $5.75 \pm 0.36^{c}$ & $5.15 \pm 0.47$ & $3.27 \pm 0.36$ & $2.67 \pm 0.48$ \\
4 & $46^{b}$ & $5.38 \pm 0.49$ & $4.01 \pm 1.33$ & $3.13 \pm 0.55$ & $2.01 \pm 0.81$ \\
\hline
\end{tabular}

Note. Triatomines fed on infected guinea pigs at weekly intervals.

${ }^{a}$ Female.

${ }^{b}$ Fifty triatomines of each stage initiated experiment; only bugs surviving 2 months were examined.

$c \overline{\mathrm{x}} \pm \mathrm{SD}$ of $\log _{10}(\mathrm{x}+1)$.

megistus to starvation was tested. We held 20 adult females and 20 fourth-stage nymphs without feeding immediately after molting. Bugs were observed daily and the mortality was recorded. Nymphs were more resistant to starvation. Ninety-five percent of adults died after 40 days of starvation and all died by 50 days. In contrast, only $5 \%$ of nymphs died by 40 days, $50 \%$ mortality was reached by 120 days, and $100 \%$ mortality at 150 days.

We examincd the influence of fecding regimes on metacyclogenesis. Fourth-stage nymphs were divided up into four groups containing 25 bugs of approximately equal weight (76-86 mg). Group 1 was fed on infected guinea pigs weekly for 8 weeks. Group 2 fed on infected guinea pigs weekly for 4 weeks and then fed during the four subsequent weeks on noninfected guinea pigs. Group 3 fed the first week on an infected guinea pig and was then fed on noninfected guinea pigs for 7 weeks. Group 4 fed the first week on an infected guinea pig and then was starved during 7 weeks. All nymphs were examined 2 months after initial bloodmeal. Group 1 contained the greatest density of flagellates and meta-
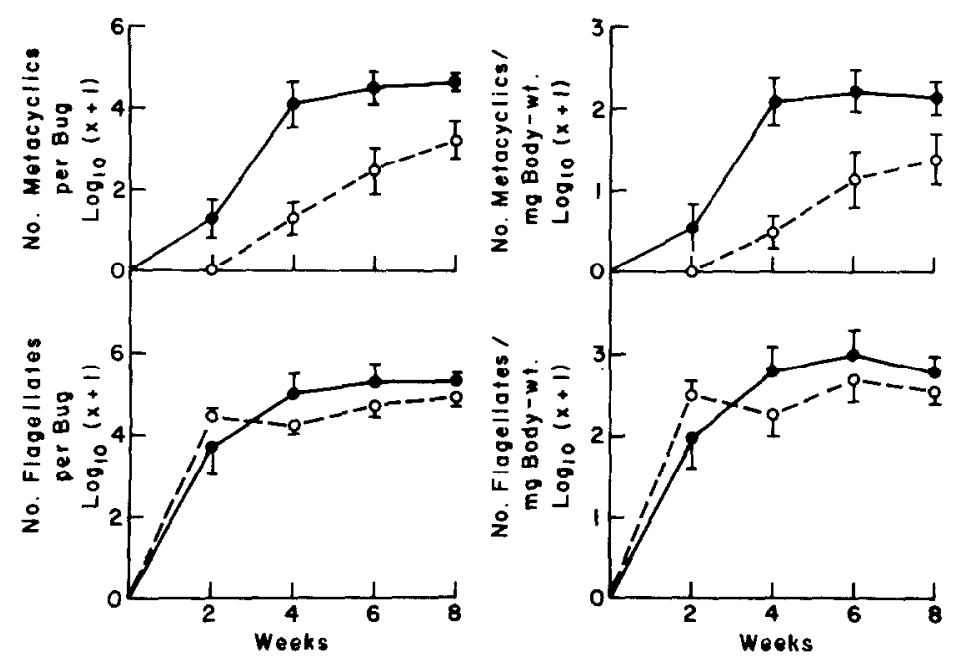

FIG. 3. Timing of differentiation to the metacyclic trypomastigote stage during Trypanosoma cruzi infection in Panstrongylus megistus. Fourth-stage nymphs and adult females fed weekly on infected guinea pigs. Twelve nymphs and twelve adult $P$. megistus were examined at biweekly intervals. Closed circles represent means of female adults, open circles represent means of fourth-stage nymphs ( $\pm \mathrm{SE}$ ). 
cyclics (Table III). However, there was little difference between Groups 1, 2, and 3. Only Group 4 contained markedly fewer flagellates and metacyclics. Group 3 contained significantly more metacyclics than Group $4(t=5.33, P<0.001)$. The provision of a bloodmeal, albeit noninfectious, was enough to stimulate metacyclogenesis in Group 3, while little metacyclogenesis occurred in the starved Group 4.

\section{DISCUSSION}

The ability to undergo metacyclogenesis may be both an inherent quality of particular Trypanosoma cruzi strains (Crane and Dvorak 1982; Garcia and Dvorak 1982) and the response to stimuli provided by triatomine organs or extracts (Wood and Souza 1976; Lanar 1979; Isola et al. 1981). Organic acids have been considered to be important in controlling metacyclogenesis (Fernandes et al. 1969). However, the addition of organic acids did not trigger metacyclogenesis in vitro (Zeledon et al. 1977; Pan et al. 1978a). Moreover, antibodies (Sher and Snary 1982) as well as lectins (Pereira et al. 1981) have been implicated in the control of metacyclogenesis. However, the effect of these agents within triatomines has not been established. Metacyclogenesis may take place only after repeated multiplication of epimastigotes (Pan 1978a, b). Perhaps, nutritional factors control metacyclogenesis (Sher et al. 1983; Ucros et al. 1983). In our study, starved triatomines did not produce large numbers of metacyclic trypomastigotes. Multiple bloodmeals could have provided the stimulus for epimastigote division and subsequent differentiation to metacyclics. In adult triatomines, the rapid rate of digestion may speed up metacyclogenesis.

Wood (1960) suggestcd that contamination with feces from adult triatomines carried greater risk of acquiring $T$. cruzi than did contamination with feces from nymphal triatomines, since nymphal feces contained few metacyclics. However, he found that

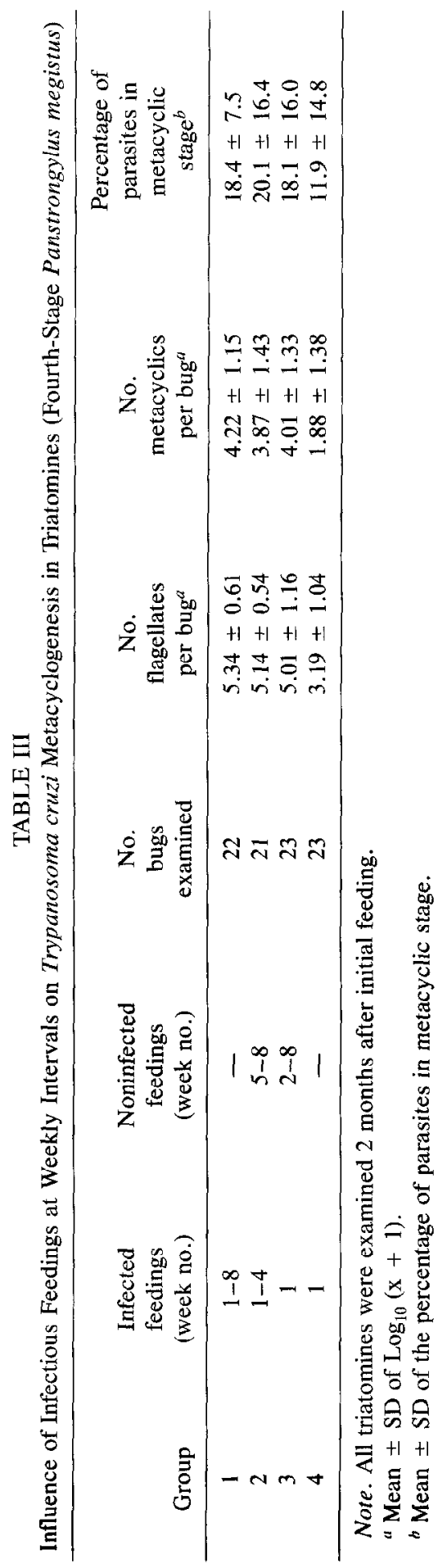


nymphs defecate more frequently than adults, making contact with nymphal feces more likely. Piesman and Sherlock (1983) observed that adult Panstrongylus megistus produced more feces than firstto fourth-stage nymphs, but fifth-stage nymphs produced the largest volume of feces. In our present study, we found naturally infected adult $P$. megistus contain $9.8 \times$ more metacyclics than fifth-stage nymphs. The increased metacyclic density in adults probably presents a greater risk of transmission than does the slightly greater volume of feces produced by fifth-stage nymphs.

Attempts to control triatomines have utilized insect growth regulators, i.e., juvenile hormone (Pinchin et al. 1978) and precocene (Tarrant and Cupp 1978). In the short run, such manipulations may increase the proportion of triatomines in the adult stage. A triatomine population so structured may increase the potential for transmission of Trypanosoma cruzi.

\section{ACKNOWLEDGMENTS}

The Harvard component, under the direction of Thomas H. Weller, is supported by the Wellcome Trust and U.S. National Institutes of Health Grant AI1630505; its collaborative activities in Brazil are under the aegis of the Pan American Health Organization. Field work was assisted by Sr. Antonio C. Batista, Sr. Antonio C. Santos, and Sr. Tome S. Oliveiro. Laboratory support was provided by Celia Lima and Maria C.B. Soares. Facilities at the Faculdade de Medicina were provided by the Federal University of Bahia, Brazil. The authors thank Dr. T. H. Weller and Dr. S. Pan for critical reading of the manuscript.

\section{REFERENCES}

BRACK, C. 1968. Elektronenmikroskopische Untersuchungen zum lebenszyklus von Trypanosoma cruzi unter besonderer Berucksichtigung der Entwicklungsformen im uebertrager Rhodnius prolixus. Acta Tropica 25, 289-356.

BRENER, Z. 1972. A new aspect of Trypanosoma cruzi life-cycle in the invertebrate host. Journal of Protozoology 19, 23-27.

BRLMPT, E. 1912. Le Trypanosoma cruzi evolve chez Conorhinus megistus, Cimex lectularius, Cimex boueti, ct Ornithodorus moubata. Cyclc evolutif dc ce parasite. Bulletin de la Societe de Pathologie exotique 5, 360-367.

CRane, M. S. J., AND DvoraK, J. A. 1982. Trypanosoma cruzi: Spontaneous transformation by a $\mathrm{Y}$ strain variant in liquid medium. Experimental Par. asitology 54, 87-92.

Dras, E. 1934. Estudos sobre o Schizotrypanum cruzi. Memorias do Instituto Oswaldo Cruz 28, 1-110.

Fernandes, J. F., Castellani, O., ANd Kimura, E. 1969. Physiolngical events in the course of the growth and differentiation of Trypanosoma cruzi. Genetics 61 (Suppl.), 214-225.

Garcta, E. S., AND DvoraK, J. A. 1982. Growth and development of two Trypanosoma cruzi clones in the arthropod Dipetalogaster maximus. American Journal of Tropical Medicine and Hygiene 31, 259262.

Hoare, C. A., And Wallace, F. G. 1966. Development stages of trypanosomatid flagellates: A new terminology. Nature (London) 212, 1385-1386.

IsOLA, E. L. D., LAMMEL, E. M., KATZIN, V. J., AND Goncalez CaPPa, S. M. 1981. Influence of organ extracts of Triatoma infestans on differentiation of Trypanosoma cruzi. Journal of Parasitology 67, 53-58.

LANAR, D. E, 1979. Growth and differentiation of Trypanosoma cruzi cultivated with a Triatoma infestans embryo cell line. Journal of Protozoology 26, 457-462.

Miles, M. A., Patterson, J. W., Marsden, P. D., AND MINTER, D. M. 1975. A comparison of Rhodnius prolixus, Triatoma infestans and Panstrongylus megistus in the xenodiagnosis of a chronic Trypanosoma (Schizotrypanum) cruzi infection in a rhesus monkey (Macaca mullatta). Transactions of the Royal Society of Tropical Medicine and Hygiene 69, 377-382.

MinTER, D. M. 1978. Triatomine bugs and the household ecology of Chagas" disease. In "Medical Entomology Centenary Symposium Proceedings, " pp. 85-93. Royal Society of Tropical Medicine and Hygiene, London.

Mott, K. E., Lehman, J. S., Hoff, R., Morrow, R. H., Muniz, T. M., Sherlock, I., Draper, C. C., Pugliese, C., And Guimaraes, A. C. 1976. The epidemiology and household distribution of seroreactivity to Trypanosoma cruzi in a rural community in northeast Brazil. American Journal of Tropical Medicine and Hygiene 25, 552-562.

MotT, K. E., Muniz, T. M., Lehman, I. S., JR., Hofr, R., Morrow, R. H., JR., Oliveira, T. S., SHERloCK, I., AND DRAPER, C. C. 1978. House construction, triatomine distribution, and household distribution of seroreactivity to Trypanosoma cruzi in a rural community in northeast Brazil. American Journal of Tropical Medicine and Hygiene 27, 1116-1122. 
Mshelbwala, A. S., and Ormerod, W. E. 1973. Measurement of the infectivity of Trypanosoma cruzi in faeces of Rhodnius by comparison of doseresponse curves. Journal of General Microbiology 75, 339-350.

NeAL, R. A., ANd McHaRdy, N. 1977. Comparison of infectivity of Trypanosoma cruzi bloodstream trypomastigotes from Rhodnius prolixus. Acta Tropica 34, 79-85.

PAN, S. C. T. 1978a. Trypanosoma cruzi: Intracellular stages grown in a cell-free medium at $37^{\circ} \mathrm{C}$. Experimental Parasitology 45, 215-224.

PAN, S. C. T. 1978b. Trypanosoma cruzi: Ultrastructure of morphogenesis in vitro and in vivo. Experimental Parasitology 46, 92-107.

Pereira, M. E. A., Andrade, A. F. B., ANd Ribeiro, J. M. C. 1981. Lectins of distinct specificity in Rhodnius prolixus interact selectively with Trypanosoma cruzi. Science (Washington, D.C.) 211, 597-600.

Perlowagora-Szumlewicz, A., and Muller, C. A. 1982. Studies in search of a suitable experimental insect model for xenodiagnosis of hosts with Chagas' disease. 1-Comparative xenodiagnosis with nine triatomine species of animals with acute infections by Trypanosoma cruzi. Memorias do Instituto Oswaldo Cruz 77, 37-53.

Piesman, J., AND Sherlock, I. A., 1983. Factors controlling the volume of feces produced by triatomine vectors of Chagas' disease. Acta Tropica 40, 351358.

Piesman, J., Sherlock, I. A., And Christensen, H. A. 1983. Host availability limits population density of Panstrongylus megistus. American Journal of Tropical Medicine and Hygiene 32, 1445-1450.

Pinchin, R., Oliveira Filho, A. M., Figueiredo, M. J., Muller, C. A., ANd Gilbert B. 1978. Slowrelease juvenile hormone formulations for triatomine control. Transactions of the Royal Society of Tropical Medicine and Hygiene 72, 322-323.

Sher, A., Crane, M. S. J,, ANd Kirchoff, L. V. 1983. Incubation in mice provides a signal for the differentiation of Trypanosoma cruzi epimastigotes to trypomastigotes. Journal of Protozoology 30 , 278-283.
Sher, A., AND SNARY D. 1982. Specific inhibition of the morphogenesis of $T$. cruzi by a monoclonal antibody. Nature (London) 300, 639-640.

TARrant, C. A., AND Cupp, E. W. 1978. Morphogenetic effects of Precocene II on the immature stages of Rhodnius prolixus. Transactions of the Royal Society of Tropical Medicine and Hygiene 72, 666668.

Torres, C. B. M. 1915. Alguns fatos que interessam a epidemiologia da molestia a Chagas. Memorias do Instituto Oswaldo Cruz 7, 120-138.

Ucros, H., Granger, B., AND Krassner, S. M. 1983. Trypanosoma cruzi effect of $\mathrm{pH}$ on in vitro formation of metacyclic trypomastigotes. Acta Tropica $40,105-112$.

Urdaneta-Morales, S., AND Rueda, I. G. 1977. A comparative study of the behavior of Venezuelan and Brazilian strains of Trypanosoma (Schizotrypanum) cruzi in the Venezuelan invertebrate host (Rhodnius prolixus). Revista do Instituto de Medicina Tropical de Sao Paulo 19, 241-250.

Wouv, S. F. 1960. A potential index for Triatoma harboring Trypanosoma cruzi Chagas. Experimental Parasitology 10, 356-365.

WoOD, D. E., AND SouzA, O. E. 1976. Trypanosoma cruzi: Effects of Rhodnius prolixus extracts on in vitro development. Revista do Instituto de Medicine Tropical de Sao Paulo 18: 93-96.

Zarate, L. G., Zarate, R. J., Tempelis, C. H., AND GoLDSMITH, R. S. 1980. The biology and behavior of Triatoma barberi (Hemiptera: Reduviidae) in Mexico. I. Blood meal sources and infection with Trypanosoma cruzi. Journal of Medical Entomology 17, 103-116.

Zeledon, R., Alvarenga, N. J., And Schosinsky, K. 1977. Ecology of Trypanosoma cruzi in the insect vector. In "Chagas Disease" (Proceedings of an International Symposium, New York, June 1977), pp. 59-70. Pan American Health Organization Scientific Publication 347, Washington, D.C.

Zeledon, R., Bolanos, R., and Rojas, M. 1984. Scanning electron microscopy of the fintal phase of the life cycle of Trypanosoms cruzi in the insect vector. Acta Tropica 41, 39-43. 